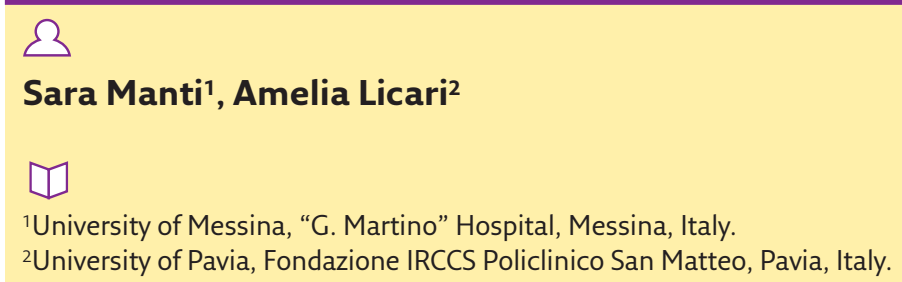

"University of Messina, "G. Martino" Hospital, Messina, Italy.

University of Pavia, Fondazione IRCCS Policlinico San Matteo, Pavia, Italy.

\title{
How to obtain informed consent for research
}

\section{Doing science}

Cite as: Manti S, Licari A. How to obtain informed consent for research. Breathe 2018; 14: 145-152.
Current biomedical research on human subjects requires clinical trial, which is defined as "any research study that prospectively assigns human participants or groups of humans to one or more health-related interventions [i.e. drugs, cells or other biological products, surgical procedures, devices] to evaluate the effects on health outcomes" [1]. In our modern ethical conception, all research conducted on humans must be pre-emptively accepted by the subjects themselves through the procedure known as informed consent, which is a process by which "a subject voluntarily confirms his or her willingness to participate in a particular trial, after having been informed of all aspects of the trial that are relevant to the subject's decision to participate", as stated in the International Council for Harmonisation Good Clinical Practice guidelines [2]. Informed consent is documented by means of a written, signed and dated informed consent form. This form is required in the following cases: 1) when the research involves patients, children, incompetent/incapacitated persons, healthy volunteers, immigrants or others (e.g. prisoners); 2) when the research uses/collects human genetic material, biological samples or personal data [3].

The informed consent form must be written in language easily understood by the subjects, it must minimise the possibility of coercion or undue influence, and the subject must be given sufficient time to consider participation. However, informed consent is not merely a form that is signed, but is a process in which the subject has an understanding of the research and its risks, and it is tightly described in ethical codes and regulations for human subject research [2].

\section{The informed consent process}

The voluntary expression of the consent by a competent subject and the adequate information disclosure about the research are critical and essential elements of the informed consent process [4]. Competent subjects able to comprehend the research-related information should personally decide and provide the consent on research participation. Conditions posing practical

\section{Educational aims}

- To provide a comprehensive overview of issues in obtaining informed consent in clinical research.

- To describe the process of obtaining informed consent in clinical trials.

- To highlight the circumstances under which informed consent can be waived.

- To review the setting of obtaining informed consent from "vulnerable populations". 
challenges in obtaining informed consent from the real subject may include situations of medical emergency or obtaining consent from "vulnerable" subjects and/or children [5].

Research-related information must be presented to enable people to voluntarily decide whether or not to participate as a research subject. For an ethically valid consent, information provided to a research subject should include, but not be limited to: information about the health condition for which the research is proposed; details of the nature and purpose of the research; the expected duration of the subject's participation; a detailed description of study treatment or intervention and of any experimental procedures (including, in the case of randomised clinical trials (RCTs), also blinding and randomisation); a statement that participation in research is voluntary; probable risks and benefits associated with research participation; details of the nature of the illness and possible outcome if the condition is left untreated; availability, risks and benefits of alternative treatments; information about procedures adopted for ensuring data protection/confidentiality/privacy, including duration of storage of personal data; details about the handling of any incidental findings of the research; description of any planned genetic tests; details of insurance coverage in case of injury; reference contacts for any further answers to pertinent questions about the research and the subject's rights and in case of any research-related injury to the subject; and any other information that seems necessary for an informed decision to be taken by the subject. Of particular importance, a statement offering the subject the opportunity to withdraw at any time from the research without consequences must be provided during the information disclosure [2]. Specific information should be provided in case of research projects involving children, incapacitated adults not able to give informed consent, illiterate populations, etc. (as will be described later in this article).

The information about the research should be given by a physician or by other individuals (i.e. researchers) with appropriate scientific training and qualifications [6]. Furthermore, the location where the informed consent is being discussed, and the subject's physical, emotional and psychological capability, must be taken into consideration when taking consent from a human subject.

Table 1 Conditions to be met in emergency clinical study

- Subjects affected by a life-threatening condition

- The treatment is experimental

- The clinical research allows verification of both the effectiveness and safety of the treatment

- It is impracticable to obtain consent

- The waiver of informed consent is needed for the clinical trial

- The researcher will contact the legally authorised representative

- The family members can decline the patient's participation in the study

\section{Informed consent: when is it not necessary?}

After institutional review board (IRB) or independent ethics committee approval is achieved, obtaining informed consent from each human subject prior to his/her participation in clinical trial is mandatory [5]. However, when specific circumstances occur, the informed consent can be waived, and "research without consent" is possible, which allows enrolment of patients without their consent, under strict regulation [7]. In order that research without consent is considered justifiable, the following three conditions have to be met: 1 ) it is impracticable to obtain consent, 2) the research does not infringe the principle of self-determination, and 3) the research provides significant clinical relevance [8].

The first condition, of "impracticability", occurs when obtaining informed consent is burdened by high impact in terms of time and economic resources or could compromise the study's validity [8]. The second condition means that, although physicians are requested to ensure that the patient has understood the aim of the research and the risks and/or benefits associated with study participation, the researchers are also advised to respect the patient's decision-making capacity, not interfering with his/her decisions and acting always in the patient's best interest [9]. The third condition leads to justification of waiving consent when the clinical relevance and public health importance are potentially high [8].

The formal literature identifies different types of RCTs and classifies them into three macro-areas: 1) RCTs based on infeasibility of informed consent; 2) RCTs that omit informed consent only for control groups; and 3) RCTs that omit informed consent entirely.

\section{RCTs based on infeasibility of informed consent}

Emergency clinical studies, involving critically ill subjects, represent an exception to the requirement of informed consent. The investigated life-saving therapy and the medical intervention may be required immediately, not permitting the researchers to wait and respect all procedures of obtaining informed consent. Within this context, the researchers will be able to proceed with patient recruitment, also without the subject's consent to treatment, when, prior to the study, the IRB has ascertained the presence of mandatory conditions (table 1) [10].

Cluster randomised studies include clustercluster and individual-cluster research [11]. In cluster-cluster designs (e.g. studies on infectious disease prevention), the intervention involves the entire target community, so that single subjects cannot refuse it [12]. Conversely, in individualcluster designs (e.g. studies on primary care), although the intervention involves all the selected 
community, the right to refuse treatment is allowed. Under this circumstance, the omission of informed consent is justified only when the treatment refusal undermines the validity of the research study and/ or procedures [13].

\section{RCTs that omit informed consent only for control groups}

In Zelen's single-consent model (e.g. RCTs in infectious or oncological diseases), randomisation occurs prior to any consent, and informed consent is sought only from individuals assigned to experimental treatment [14]. In the control group, the physicians do not make substantial changes in routine patient care, so informed consent is not required for patient enrolment [8].

In order to improve study recruitment, Zelen developed the double-consent design. Specifically, informed consent is requested for subjects to be involved in the study but not for the randomisation, preventing psychological distress [14].

In follow-up studies, the nested consent model (e.g. for single cohort studies) or cohort multiple RCTs model (e.g. for multiple cohort studies) is applied. In these variants, patients give their consent for prospective follow-up; however, they remain blinded to any randomised experimental interventions [15].

In trials using the model of "consent to postponed information", the informed consent process is carried out after the study is completed [16].

All these RCT types aim to avoid unnecessary stress in patients who will not receive the new promising experimental treatment. Moreover, these clinical study designs do not affect the standard therapeutic approach or infringe the rights of the patients in the control group; therefore, the clinical trial can proceed without obtaining informed consent [8].

\section{RCTs that omit informed consent entirely}

Based on the fact that patients are assigned to standard care interventions, no informed consent is sought either in low-risk pragmatic RCTs [17] or in prompted optional randomisation trials [18, 19]. However, in a low-risk pragmatic RCT, patients do not have the possibility to choose one of the two standard treatments, whereas in a prompted optional randomisation trial, both the researchers and the enrolled patients can choose one type of treatment over another, despite the randomisation results [6].

\section{Special needs: vulnerable patients}

A "vulnerable population" is defined as a disadvantaged community subgroup unable to make informed choices, protect themselves from inherent or intended risks, or keep their own interests safeguarded [20]. In the health domain, "vulnerable populations" refers to physical vulnerability (e.g. pregnant women, fetuses, children, orphans, students, employees, prisoners, the military, and those who are chronically or terminally ill), psychological vulnerability (cognitively and intellectually impaired individuals) and social vulnerability (those who are homeless, from ethnic minorities, are immigrants or refugees) [20].

Due to a compromised free will and inability to make conscious decisions, several ethical dilemmas (related to communications, privacy and treatment) often arise when research involves these populations. Guaranteeing protection of rights, safety, data privacy and confidentiality of vulnerable subjects are prerogatives of good clinical practice, and law dispositions are regulated and strictly monitored by the applicable authorities [21].

\section{Physical vulnerability}

For a long time, pregnant women were excluded from clinical research because of their "vulnerability". Although pregnant women are able to make informed and conscious choices, they have been considered "vulnerable" due to the potential risks to the fetus, who is also considered as a "patient" [22]. More recently, with the consideration of pregnant women as "scientifically complex" rather than "vulnerable" subjects, it has been permitted to involve this category in research trials [23]. The "scientific complexity" reflects both ethical and physiological complexity. The ethical aspects are secondary to the need to find a balance between interests of the fetus and the mother. The physiological aspects are strictly related to the pregnancy status [24].

Research studies involving pregnant women and fetuses have to satisfy specific federal regulations (table 2). The following appropriate precautions

Table 2 Conditions to be met in research studies involving pregnant women and fetuses

- In vivo studies have also been conducted on pregnant animals

- Clinical studies have been conducted on nonpregnant women

- Clinical findings assessing potential harms to pregnant women and fetuses are available

- The risk to the fetus is minimal and caused exclusively by the procedure/intervention

- The study will achieve crucial knowledge not obtainable by any other means

- The researchers will have no part in any decision influencing fetal viability or pregnancy

- No incentive will be provided to influence the course of pregnancy 
should be taken in research studies involving pregnant women: no pregnant woman may be involved as a subject in a human clinical research project unless the purpose of the research is to meet the health needs of the mother and the fetus will be placed at risk only to the minimum extent necessary to meet such needs, or the risk to the fetus is minimal [25].

Researchers can enrol pregnant women only when the mother and/or the father are legally competent. In fact, the consent to participate in research may be either self-directed (only the mother's consent is required) or made with the guidance of the woman's partner. However, the father's consent need not be obtained when: 1 ) the research activity is directed to the health needs of the mother; 2 ) the father's identity is doubtful; 3) the father is absent; or 4) a pregnancy from rape has occurred [26]. The consent signature requirements from the mother and father are summarised in table 3 . Once the informed consent is obtained, the pregnant women will be included into any phase of the study unless the research project will be compromised or the patient's health (mother and/or fetus) will be in danger.

Medical students and employees, who take part in numerous aspects of patient care in primary, secondary and tertiary care settings, are often invited to participate in human studies as volunteers. Frequently, the requesting researcher is their supervisor or instructor, who may push them to participate in the study, which can negatively influence their decision and also violate the consent legitimacy. Therefore, in order to protect these subjects against "coercion" or "undue influence", when an investigator wishes to recruit medical students or employees, they must first obtain IRB approval for inclusion in the study of these vulnerable subgroups [27].

Prisoners, defined as any individual involuntarily confined or detained in a penal institution, are considered as "vulnerable" because they may be coerced into study participation, and also, due to both cognitive and psychiatric disorders, they can show an impaired ability to provide voluntary informed consent [28]. To protect this population, the Office for Human Research Protections has stipulated federal regulations according to which the only studies that may involve prisoners are those with independent and valid reasons for involving them (table 4) [25].

Due to the context of war in which they work, as well as the critical care setting in which they are treated, military subjects often receive medical care and/or participate in biomedical research under an "implied consent" condition. Moreover, the superior-subordinate relationship contributes to favour coercion or undue influence, making this population vulnerable [29]. To curb this

Table 3 Consent signature requirements for pregnant women and children

\section{For pregnant women}

Direct benefit to mother

Direct benefit to mother and fetus

Direct benefit to fetus

\section{For children}

Direct benefit to individual subjects

No direct benefit to individual subjects

No direct benefit to the subject or societal (indirect) benefit

Medical care related to pregnancy

Medical care related to mental health treatment, or the diagnosis or treatment of infectious, contagious or communicable diseases

Self-sufficient minors

Aged $\geq 15$ years

Living alone

Managing their own financial affairs

Emancipated minors

Married or divorced

On active duty in the US armed forces

By a court

Having the legal right to consent on their own behalf to medical, dental or mental health treatment
Mother

Mother

Mother and father

One parent or guardian ${ }^{\#}$

Both parents

Both parents

Parental consent is not needed

Parental consent is not needed

Parental consent is not needed

Parental consent is not needed

\#: consent requirements are the same whether the risk is "no more than minimal" or "more than minimal". 
phenomenon and to ensure that participation is truly voluntary, the US Dept of Defense agencies have adopted requirements similar to those that govern medical research that applies to the civilian population. Accordingly, the medical research recruitment session happens in the absence of superiors, and the informed consent is obtained prior to participating in a medical research study. The presence of an ombudsman guarantees and verifies that the participation is voluntary and that the information provided during recruitment is complete, accurate and clear. A payment as an incentive is acceptable but it must not be used to legitimise a coercive interference. Additional protection is provided to students at service academies, especially those aged $<18$ years. However, when emergency research is conducted or the research study advances the development of a medical product needed by the armed forces, informed consent will not be required [29].

\section{Psychological vulnerability}

Mental disability may compromise the selfdetermination and decision-making capacities [30]. Researchers interested in enrolling individuals with cognitive disorders are invited to apply different strategies to promote a better understanding of information-gathering processes. Simplifying the questions and content, adopting supportive technologies, using a more simple language, and spending more time for the information process have been suggested as useful and valid measures. When all these strategies prove to be insufficient, the investigators are required to obtain consent from a legally authorised representative [30].

\section{Social vulnerability}

Similarly to other vulnerable populations, research involving the homeless, ethnic minorities, immigrants and refugees is regulated by laws and specific procedures. Cultural and language differences, "undocumented" migrant status, and the precarious legal positions of these subjects raise several ethical issues, such as whether the participation is truly voluntary, or there are unrealistic expectations, or any benefits for their "status".

Obtaining informed consent in these groups is extremely complex. A friendly procedure has been identified as the best way to adequately involve these vulnerable groups. A health centre or community building could represent an accessible location. The reimbursement of travel expenses for applicants can be a valid solution to obtain a representative sample for the clinical research. Clear and simple language, emphasising confidentiality, with the help of professional interpreters, can tempt migrants to sign the consent form. Lastly, the possibility of receiving something back in return for their contribution may enable successful enrolment of migrants in research [31].
Table 4 Studies that may involve prisoners

- Studies on the possible causes, processes and effects of incarceration

- Studies on prisons as institutional structures or on prisoners as incarcerated persons

- Studies on special conditions affecting prisoners

- Studies on practices of improving the health or well-being of the prisoners

- Epidemiological studies

\section{Special needs: children}

Because of their young age as well as their limited emotional and intellectual abilities, children are considered to be legally incompetent to give valid informed consent; thus, to enrol a child in a research study, the permission by at least one parent or legal representative is mandatory (table 3 ). For subjects aged $<18$ years, biological or adoptive parents or legal guardians (persons having both legal capacity and responsibility) can give consent on behalf of their child, exercising free power of choice without any form of coercion. While married mothers and fathers both have parental responsibility, unmarried parents can exert parental responsibility only if they are named individually on the child's birth certificate. Also, divorced parents maintain parental responsibility, but it is necessary to know to whom the child's custody has been assigned [32]. However, on this matter, the European laws and regulations are not harmonised and several discrepancies are present in each country [33].

Despite potential benefits for the research subjects, the failure of parents to give consent (or their refusal to give consent) is not a rare circumstance [34]. It can be the case that

\section{Self-evaluation questions}

1. Which of the following pieces of information need not be included when obtaining informed consent?
a) Diagnosis
b) Risks and benefits of treatment
c) Alternatives to treatment
d) Family's wishes

2. In which of the following circumstances could informed consent be waived?

a) When a minor is considered as emancipated

b) When a patient is found to be incompetent

c) When immediate treatment is necessary to prevent death or permanent impairment

d) When the subject is aged $>18$ years

3. In which of the following cases is a minor not considered emancipated or self-sufficient?

a) Minor is married or divorced

b) Minor on active duty in the US armed forces

c) Minor is considered self-sufficient by a court

d) Minor having a son 


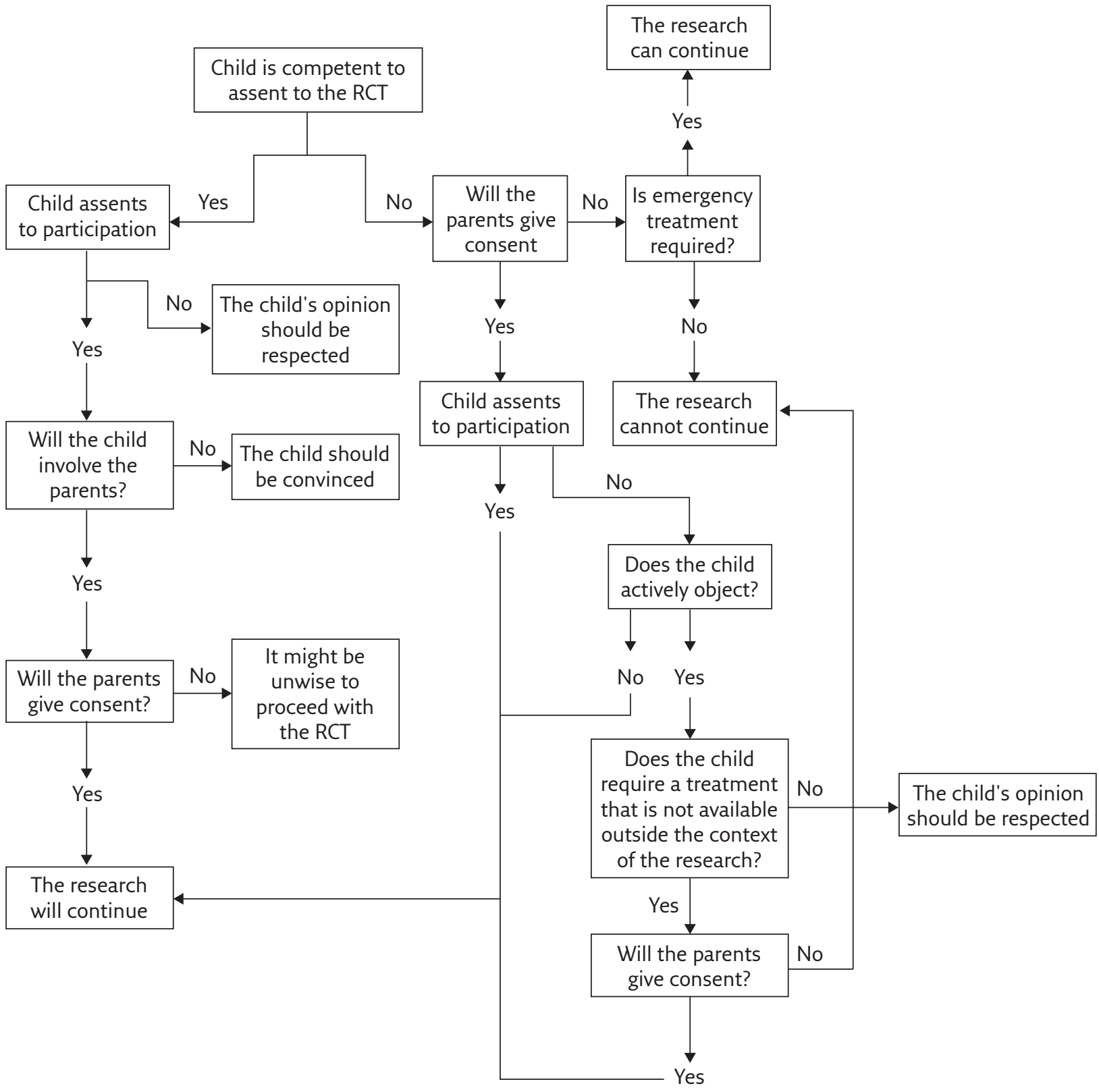

Figure 1 Flow chart of parental permission requirements.

researchers are dealing with underage parents, so that, although underage parents are responsible for representing their children, as minors themselves they are not considered to be sufficiently mature; therefore, they will be not able to give valid consent. Literacy and socioeconomic levels have been identified as the most common reasons for parental non-response [34]. Clarity and adequate explanation of research information materials should be part of effective planning to overcome language and social barriers.

In clinical studies in which the adopted methodology constitutes "less than minimal risks" for children, passive parental consent represents a possible way to more easily obtain informed parental consent [34]. Furthermore, parents can be informed with regard to a possible study involving their children, and, at the time of data collection, only the child's assent is required. In fact, although the child's decision-making capacity and understanding of the research project in which he/she will be involved may be limited, the Medical Research Council have shown that, when study details are provided and communicated in a clear and adequate manner, the child can be able to reach a decision and participate consciously in the research [35]. "Assent" is the term coined to express the child's willingness to participate in clinical trials despite their young age. The "assent" should include and respect the following key points: 1) helping the child to acquire disease awareness; 2) explaining the potential impact 


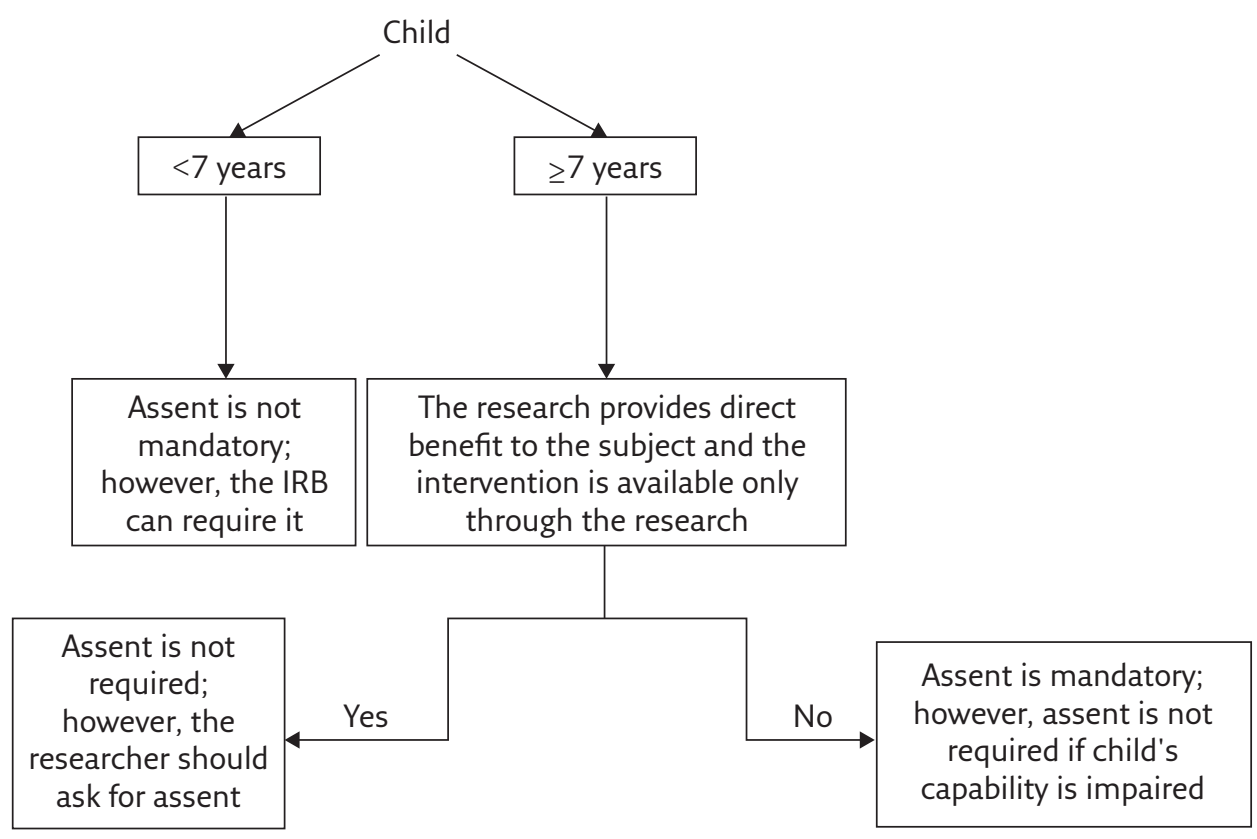

Figure 2 Flow chart of child assent requirements.

of the experimental treatment; 3) evaluating the child's ability to understand and adapt to new situations or challenges; and 4) positively influencing the patient's willingness to participate in clinical trials [36]. Although the "assent" is not mandatory for research offering a direct benefit for the child, it arises from the need to respect paediatric research subjects [37]. The evaluation of the capacity to provide the "assent" is based on developmental stage, intellectual abilities and life or disease experience. Usually, the cut-off age of 7 years is used for the beginning of logical thought processes and rational decision making [38]. However, "assent" for children aged $<7$ years can be also required once the ability to read and write has been verified [32]. Figures 1 and 2 summarise the parental and assent permission requirements, respectively.

\section{Summary}

When conducting clinical research, the obtaining of informed consent is required. Informed consent is a procedure through which a competent subject, after having received and understood all the researchrelated information, can voluntarily provide his or her willingness to participate in a clinical trial. However, when it is impracticable to obtain consent, and the research does not infringe the principle of self-determination and also provides significant clinical relevance, the researcher is legally authorised to proceed without informed consent. Furthermore, in order to preserve the selfdetermination and decision-making rights, specific law dispositions are applied when vulnerable populations are enrolled in clinical trials.

\section{Key points}

- All research conducted on humans must be pre-emptively accepted by the subjects themselves through the procedure known as informed consent.

- Voluntary expression of consent and adequate information disclosure about the research are critical and essential elements of the informed consent process.

- When specific circumstances occur, informed consent can be waived: if it is impracticable to obtain consent, if the research does not infringe the principle of self-determination, and if the research provides significant clinical relevance.

- Participation of vulnerable patients in clinical trials is regulated by specific law dispositions.

\section{Conflict of interest}

None declared. 


\section{Suggested answers}

1. d.

2. c.

3. d.

\section{References}

1. World Health Organization. Clinical Trials. www.who.int/topics/ clinical_trials/en/Date last accessed: March 22, 2018.

2. International Council for Harmonisation of Technical Requirements for Pharmaceuticals for Human Use (ICH). Integrated addendum to ICH E6(R1): Guideline for Good Clinical Practice E6(R2). Current Step 4 version. Available from: www.ich.org/products/guidelines/efficacy/article/efficacyguidelines.html Date last updated: November 9, 2016. Date last accessed: March 22, 2018.

3. European Commission. Ethical Review in FP7. Guidance for Applicants: Informed Consent. 2013. http://ec.europa. eu/research/participants/data/ref/fp7/89807/ informed-consent_en.pdf

4. Nardini $C$. The ethics of clinical trials. Ecancermedicalscience 2014; 8: 387

5. Gupta UC. Informed consent in clinical research: revisiting few concepts and areas. Perspect Clin Res 2013; 4: 26-32.

6. World Medical Association. WMA Declaration of Helsinki Ethical Principles for Medical Research Involving Human Subjects. 59th WMA General Assembly, Seoul, Korea 2008. Available from: www.wma.net/policies-post/wmadeclaration-of-helsinki-ethical-principles-for-medicalresearch-involving-human-subjects/

7. Lecouturier J, Rodgers H, Ford GA, et al. Clinical research without consent in adults in the emergency setting: a review of patient and public views. BMC Med Ethics 2008; 9: 9.

8. Gelinas L, Wertheimer A, Miller FG. When and why is research without consent permissible? Hastings Cent Rep 2016; 46: 35-43.

9. Veterans Health Administration. VHA Handbook 1004.1. Informed Consent for Clinical Treatments and Procedures. 2009. Available from: www.va.gov/VHAPUBLICATIONS/ ViewPublication.asp?pub_ID=2055 Date last updated: September 20, 2017. Date last accessed: March 22, 2018.

10. US Dept of Health and Human Services. Guidance for Institutional Review Boards, Clinical Investigators, and Sponsors: Exception from Informed Consent Requirements for Emergency Research. 2013. www.fda.gov/downloads/ regulatoryinformation/guidances/ucm249673.pdf

11. Sim J, Dawson A. Informed consent and clusterrandomized trials. Am J Public Health 2012; 102: 480-485.

12. Vanlerberghe $\mathrm{V}$, Toledo $\mathrm{ME}$, Rodríguez $\mathrm{M}$, et al. Community involvement in dengue vector control: cluster randomised trial. BMJ 2009; 338: b1959.

13. Kumar V, Mohanty S, Kumar A, et al. Effect of community-based behaviour change management on neonatal mortality in Shivgarh, Uttar Pradesh, India: a clusterrandomised controlled trial. Lancet 2008; 372: 1151-1162.

14. Zelen M. Randomized consent designs for clinical trials: an update. Stat Med 1990; 9: 645-656.

15. Hamajima N, Yuasa H, Nakamura M, et al. Nested consent design for clinical trials. Jpn J Clin Oncol 1998; 28: 329-332.

16. Boter $\mathrm{H}$, van Delden JJ, de Haan RJ, et al. Modified informed consent procedure: consent to postponed information. BMJ 2003; 327: 284-285

17. Truog RD, Robinson W, Randolph A, et al. Is informed consent always necessary for randomized, controlled trials? N EnglJ Med 1999; 340: 804-807.

18. Flory J, Karlawish J. The prompted optional randomization trial: a new design for comparative effectiveness research. Am J Public Health 2012; 102: e8-e10.

19. Flory JH, Mushlin Al, Goodman ZI. Proposals to conduct randomized controlled trials without informed consent: a narrative review. J Gen Intern Med 2016; 31: 1511-1518.

20. Aday LA. Who are the vulnerable? In: At Risk in America: The Health and Health Care Needs of Vulnerable Populations in the United States. 2nd Edn. San Francisco, Jossey-Bass, 2001; pp. 1-15.

21. US Dept of Health and Human Services. Guidance for Clinical Trial Sponsors: Establishment and Operation of Clinical Trial Data Monitoring Committees. 2006. www.fda.gov/downloads/ regulatoryinformation/guidances/ucm127073.pdf

22. Lupton MG, Williams DJ. The ethics of research on pregnant women: is maternal consent sufficient? BJOG 2004; 111: 1307-1312.

23. Blehar MC, Spong C, Grady C, et al. Enrolling pregnant women: issues in clinical research. Womens Health Issues 2013; 23: e39-e45.

24. McCullough LB, Coverdale JH, Chervenak FA. A comprehensive ethical framework for responsibly designing and conducting pharmacologic research that involves pregnant women. Am J Obstet Gynecol 2005; 193: Suppl. 1, 901-907.

25. Office for Human Research Protections. Code of Federal Regulations. Title 45. Public Welfare. Department of Health and Human Services. Part 46. Protection of Human Subjects. www.hhs.gov/ohrp/regulations-and-policy/ regulations/45-cfr-46/index.html Date last updated: January 15, 2009. Date last accessed: March 22, 2018.

26. Spencer SA, Dawson A. Implications of informed consent for obstetric research. Obstet Gynaecol 2004; 6: 163-167.

27. Ravindran RP, Lester DG, Nawab KA, et al. The issue of "informed consent" in medical student introductions. Perspect Med Educ 2015; 4: 100-101.

28. Moser DJ, Arndt S, Kanz JE, et al. Coercion and informed consent in research involving prisoners. Compr Psychiatry 2004; 45: 1-9.

29. McManus J, Mehta SG, McClinton AR, et al. Informed consent and ethical issues in military medical research. Acad Emerg Med 2005; 12: 1120-1126.

30. Wark S, MacPhail C, McKay K, et al. Informed consent in a vulnerable population group: supporting individuals aging with intellectual disability to participate in developing their own health and support programs. Aust Health Rev 2017; 41 436-442.

31. van den Muijsenbergh $M$, Teunissen $E$, van WeelBaumgarten E, et al. Giving voice to the voiceless: how to involve vulnerable migrants in healthcare research. $\mathrm{Br} J \mathrm{Gen}$ Pract 2016; 66: 284-285.

32. Cvetkovic Muntañola $A$. The essentials of the informed consent and assent process in paediatric clinical trials. J Clin Stud 2016; 8: 16-17.

33. Lepola P, Needham A, Mendum J, et al. Informed consent for paediatric clinical trials in Europe. Arch Dis Child 2016; 101: 1017-1025.

34. Meaux JB, Bell PL. Balancing recruitment and protection: children as research subjects. Issues Compr Pediatr Nurs 2001; 24: 241-251.

35. Medical Research Council. MRC Ethics Guide: Medical Research Involving Children. 2004. www.mrc.ac.uk/ documents/pdf/medical-research-involving-children/

36. Katz AL, Webb SA, Committee on Bioethics. Informed consent in decision-making in pediatric practice. Pediatrics 2016; 138: e20161485.

37. Simar MR, Johnson VA. Pediatric informed consent: challenges for investigators. Appl Clin Trials 2002; 11: 46-56.

38. Informed consent, parental permission, and assent in pediatric practice. Committee on Bioethics. Pediatrics 1995; 95: 314-317. 